\title{
Estratégias e desafios enfrentados por profissionais das técnicas radiológicas no atendimento ao paciente surdo
}

Strategies and challenges faced by professionals of radiological techniques in the health care of deaf patients

Estrategias y retos de los professionales de las técnicas radiológicas em la assistencia del paciente sordo

Recebido: 01/12/2021 | Revisado: 06/12/2021 | Aceito: 12/12/2021 | Publicado: 20/12/2021

\author{
Beatriz Duarte Araújo Rocha \\ ORCID: https://orcid.org/0000-0003-4506-5222 \\ Instituto Federal do Piauí, Brasil \\ E-mail: biaduarte0302@gmail.com \\ Idna de Carvalho Barros Taumaturgo \\ ORCID: https://orcid.org/0000-0001-8293-543X \\ Instituto Federal do Piaú, Brasil \\ E-mail: idnabarros@gmail.com \\ Cinthia Pires Guimarães \\ ORCID: https://orcid.org/0000-0002-8848-5385 \\ Instituto Federal do Piauí, Brasil \\ E-mail: pirescinthia124@gmail.com
}

\begin{abstract}
Resumo
O acesso a serviços essenciais à vida pela comunidade surda é um grande desafio na vida dos mesmos, devido a dificuldade comunicativa encontrada nesses ambientes. O serviço de saúde é um dos principais, por ser um serviço que a comunicação se faz indispensável para o sucesso do atendimento. Devido a isso, o objetivo da pesquisa é conhecer como se dá a comunicação entre pacientes surdos e o profissional das técnicas radiológicas durante a realização de exames de imagem e terapias radiológicas. A partir disso foi realizado um estudo de caráter descritivo com abordagem quantitativa, envolvendo 82 profissionais das técnicas radiológicas, que foram entrevistados entre 18/05/2021 à 28/05/2021 pela plataforma Google Forms, de acordo com os preceitos da Resolução 466/12. Os profissionais entrevistados são maiores de 18 anos e já haviam realizado atendimento de saúde a paciente surdos durante o exercício da sua profissão. Os resultados obtidos apontaram que a comunicação entre esse profissional e paciente surdo é realmente uma barreira comunicativa, mas que é superada a partir da utilização de estratégias de comunicação do tipo espaço-visual, e que a maioria dos profissionais das técnicas radiológicas acreditam na necessidade de capacitação em Libras, a fim de oferecer um melhor atendimento. Por fim, é possível concluir que a partir da utilização de diversos métodos bem como do auxílio do acompanhante, a maioria dos exames de imagem/terapias radiológicas foram realizadas com êxito. Porém, esse resultado não exclui a necessidade de capacitação em Libras, indispensável para melhor eficiência no atendimento ao surdo.
\end{abstract}

Palavras-chave: Comunicação efetiva; Pacientes surdos; Profissionais das técnicas radiológicas.

\begin{abstract}
The access to services essential to life by the deaf community is a great challenge in their lives, due to the communication difficulties found in these environments. The health service is one of the main ones, as it is a service that communication is essential for the success of the service. Because of this, the objective of the research is to know how communication between deaf patients and the professional of radiological techniques takes place during imaging exams and radiological therapies. From this, a descriptive study with a quantitative approach was carried out, involving 82 professionals of radiological techniques, who were interviewed between 05/18/2021 to 05/28/2021 by the Google Forms platform, in accordance with the precepts of Resolution 466/ 12. The professionals interviewed are over 18 years old and had already provided healthcare to deaf patients during their profession. The results obtained showed that communication between this professional and deaf patient is really a communicative barrier, but that it is overcome through the use of space-visual communication strategies, and that most professionals in radiology techniques believe in the need for training in Libras, in order to offer better service. Finally, it is possible to conclude that from the use of several methods as well as the assistance of the companion, most imaging tests/radiological therapies were successfully performed. However, this result does not exclude the need for training in Libras, which is essential for better efficiency in caring for the deaf.
\end{abstract}

Keywords: Effective communication; Deaf patients; Professionals of radiological techniques. 


\section{Resumen}

El acceso a los servicios esenciales para la vida de la comunidad sorda es un gran desafío en sus vidas, debido a las dificultades de comunicación que se encuentran en estos entornos. El servicio de salud es uno de los principales, ya que es un servicio cuya comunicación es fundamental para el éxito del servicio. Por ello, el objetivo de la investigación es conocer cómo se produce la comunicación entre el paciente sordo y el profesional de las técnicas radiológicas durante los exámenes de imagen y las terapias radiológicas. A partir de esto, se realizó un estudio descriptivo con abordaje cuantitativo, involucrando a 82 profesionales de técnicas radiológicas, quienes fueron entrevistados entre el 18/05/2021 al 28/05/2021 por la plataforma Google Forms, de acuerdo con los preceptos de la Resolución 466/12. Los profesionales entrevistados son mayores de 18 años y ya habían prestado asistencia sanitaria a pacientes sordos durante su profesión. Los resultados obtenidos mostraron que la comunicación entre este profesional y el paciente sordo es realmente una barrera comunicativa, pero que se supera mediante el uso de estrategias de comunicación espacio-visual, y que la mayoría de los profesionales en técnicas de radiología cree en la necesidad de una formación en Libras, en para ofrecer un mejor servicio. Finalmente, es posible concluir que a partir del uso de varios métodos así como de la asistencia del acompañante, la mayoría de las pruebas de imagen/terapias radiológicas se realizaron con éxito. Sin embargo, este resultado no excluye la necesidad de entrenamiento en Libra, que es esencial para una mejor eficiencia en el cuidado de los sordos.

Palabras clave: Comunicación efectiva; Pacientes sordos; Profesionales de técnicas radiológicas.

\section{Introdução}

Segundo dados do último censo demográfico, realizado em 2010 pelo Instituto Brasileiro de Geografia e Estatística (IBGE), existem no Brasil aproximadamente 9,7 milhões de pessoas surdas, dentre elas, mais de 2 milhões possuem grau de surdez severa. A perda auditiva afeta o principal meio de comunicação existente na nossa sociedade, a comunicação oralauditiva, resultando em desafios de inclusão social, principalmente no atendimento de serviços essenciais à vida. O atendimento de saúde se destaca nesse quesito por ser um ambiente onde a comunicação é primordial para a qualidade do serviço prestado, do diagnóstico e tratamento de todo e qualquer paciente (Britto \& Samperiz, 2010. Gatto \& Tochetto, 2007. Araujo et al., 2019).

No viés da dificuldade comunicativa entre a população surda e os profissionais das técnicas radiológicas, o acesso a esses serviços pelos surdos se faz de forma bem menos frequente, geralmente em situações de urgência ou emergência. Dessa forma, essas pessoas se tornam mais vulneráveis ao aparecimento de doenças que poderiam ter sido evitadas caso a ida aos serviços de saúde fosse mais assídua. Além disso, os surdos estão suscetíveis a busca e recebimento de informações sobre a sua saúde que podem ser falsas e acabarem por prejudicar ainda mais o estado de saúde dessas pessoas (Silva et al., 2021).

Através de um diálogo eficiente, torna-se possível interagir, acolher e conquistar a confiança dos pacientes, fatores indispensáveis para oferecer atendimento eficaz e seguro. Todavia, no que tange as pessoas com surdez, a comunicação é na verdade um desafio para o sucesso do atendimento de saúde. Devido a incompatibilidade comunicativa entre profissional da saúde, dentre eles os profissionais das técnicas radiológicas, e paciente surdo, outras formas de comunicação são utilizadas a fim de gerar o mínimo de entendimento entre profissional e paciente (Chaveiro, Barbosa \& Porto, 2008. Pereira et al., 2020. Tedesco \& Junges, 2013).

A relação dos profissionais das técnicas radiológicas com pacientes surdos deve ser estabelecida através da modalidade de comunicação espaço-visual, onde a comunicação é produzida pelo movimento das mãos, captada e decodificada pelos olhos, sendo a Língua Brasileira de Sinais (Libras) o método mais adequado, pois é a língua mais utilizada pelas pessoas surdas no Brasil. Além da Libras, outras estratégias podem ser utilizadas por esses profissionais para estabelecer comunicação efetiva com a comunidade surda, como: leitura labial, gestos, mímica, auxílio de um intérprete ou acompanhante, expressão corporal e até mesmo escrita, caso o paciente saiba ler (Chaveiro, Barbosa \& Porto, 2008. Magrini \& Santos, 2014).

Tendo em vista que para se comunicar com os pacientes surdos, os profissionais das técnicas radiológicas encontram diversos desafios e que utilizam diferentes métodos e estratégias para estabeleceram contato com esses pacientes, a fim de oferecer um atendimento de qualidade e um diagnóstico/terapia preciso, emerge o problema levantado pelo estudo: Como se dá 
a comunicação entre pacientes surdos e o profissional das técnicas radiológicas durante a realização de exames de imagem e terapias radiológicas? Como os profissionais das técnicas radiológicas promovem atendimento aos pacientes surdos?

O objetivo geral da pesquisa é conhecer como se dá a comunicação entre pacientes surdos e o profissional das técnicas radiológicas durante a realização de exames de imagem e terapias radiológicas. Os objetivos específicos são: descrever como ocorre o processo de comunicação entre o profissional e o paciente surdo durante a realização de exames de imagem e terapias radiológicas, investigar os principais desafios encontradas pelos profissionais das técnicas radiológicas para o atendimento do paciente surdo e por fim descrever, na perspectiva do profissional, como ele avalia o nível de eficiência da comunicação utilizada por ele no atendimento ao surdo.

\section{Metodologia}

Estudo de natureza descritiva com abordagem quantitativa. Segundo Prodanov \& Freitas (2013, p. 52) a pesquisa descritiva tem como atributo descrever características e/ou fenômenos que ocorrem dentro de uma população, como também estabelecer relação entre variáveis da sociedade sem que haja interferência do pesquisador, podendo ser realizada sob forma de questionário ou observação sistêmica.

A pesquisa foi aprovada pelo Comitê de Ética e Pesquisa do Instituto Federal de Educação, Ciência e Tecnologia do Piauí. O Certificado de Apresentação de Apreciação Ética (CAAE) da pesquisa é: 45141721.9.0000.9207.

O presente estudo foi desenvolvido no ano de 2021, no período de 18/05/2021 à 28/05/2021. A amostra utilizada para a realização do trabalho foi composta por profissionais das técnicas radiológicas, dentre eles técnicos, tecnólogos e estagiários. Esta amostra foi identificada a partir do banco de dados (telefone e e-mail) de alunos e ex-alunos do curso de Radiologia do IFPI. Os critérios de inclusão da pesquisa foram: ter idade superior a 18 anos e ter tido experiência no exercício da profissão em atendimento ao cliente surdo independentemente de estar trabalhando/estagiando na área da Radiologia no momento da coleta dos dados. A cada participante foi solicitado que indicasse pelo menos um colega de profissão para que o autor desta pesquisa entrasse em contato posteriormente, convidando-o a participar da pesquisa, caso atenda aos critérios de participação.

A coleta de dados foi realizada a partir de um questionário que consta de duas partes. A primeira parte aborda sobre questões do entrevistado, como: sexo, idade, setor de atuação, caracterização do local de trabalho e tempo de atuação na área da radiologia. Já a segunda parte é composta por questões de conhecimento específico, que abordam os desafios e estratégias utilizadas por esses profissionais quanto ao atendimento de pessoas com surdez.

O questionário foi desenvolvido na ferramenta Google Formulários, que gera um link para o acesso e resposta dos participantes, o qual foi enviado a cada participante após contato prévio por mensagem.

Para tanto, no primeiro contato com o participante da pesquisa, foi feita uma apresentação da pesquisa e na sequência questionado se o mesmo já teve experiência prévia no atendimento de paciente surdo; se a resposta for não, este profissional não se encaixa nos critérios de inclusão aqui estabelecidos e o contato se dá como encerrado. Caso a resposta seja sim, há uma explanação sobre os objetivos da pesquisa, e informações sobre o anonimato das informações ali cedidas. Após isso foi repassado o Termo de Consentimento Livre e Esclarecido (TCLE) também via Google Formulários. Com a leitura de todas as informações e caso o entrevistado aceite participar (resposta obrigatória) é dado início às perguntas do questionário.

A coleta de dados se baseou no princípio da 'saturação teórica', que consiste em uma ferramenta delimitadora da amostra, ou seja, a quantidade final de participantes. A coleta é interrompida a partir do momento que o autor da pesquisa percebe um padrão de repetição nas falas, sendo assim, não convém continuar coletando dados, visto que não irá acrescentar na reflexão teórica já alcançada a partir dos dados obtidos (Falqueto, Farias \& Hoffmann, 2018). 
Os dados quantitativos obtidos através de questões fechadas foram contabilizados e expressos em frequências absolutas e relativas. Para isso foi utilizado o software Excel, e posteriormente os gráficos e tabelas gerados foram exportados para o Word, para a construção desse artigo.

\section{Resultados e Discussão}

\subsection{Caracterização dos profissionais das técnicas radiológicas entrevistados}

No total, este estudo contou com a participação de 82 profissionais das técnicas radiológicas. Na Tabela 1, consta a caracterização dos profissionais das técnicas radiológicas entrevistados.

Tabela 1 - Caracterização dos profissionais das técnicas radiológicas entrevistados, N=82, Teresina-PI, 2021.

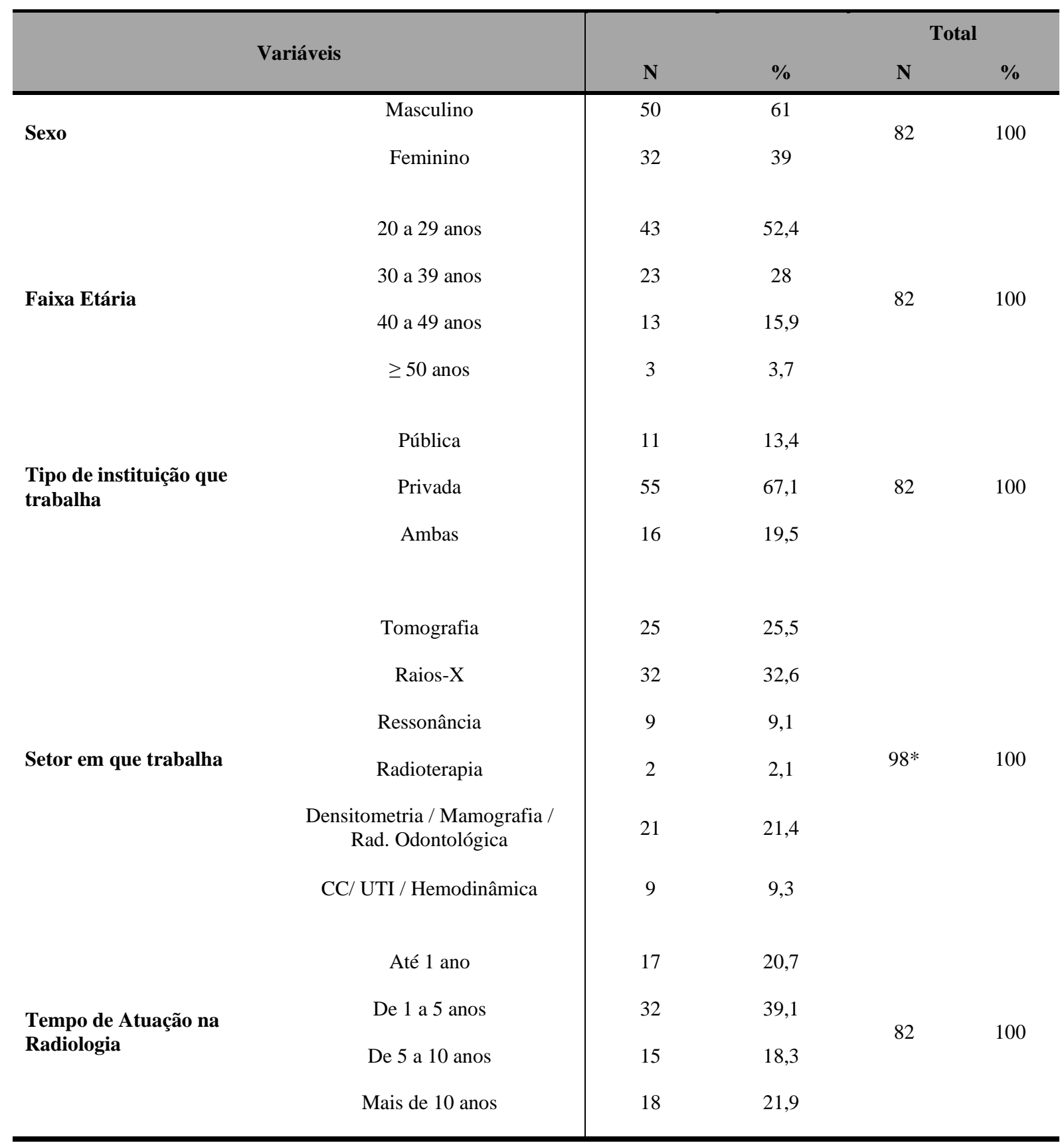


Pela Tabela 1 é possível observar que a maioria dos participantes, são indivíduos do sexo masculino (61\%); na faixa etária de 20 a 29 anos (52,4\%). Predominou profissionais que trabalham em instituição de saúde privada (67,1\%) com tempo de atuação profissional entre 1 a 5 anos $(39,1 \%)$.

Quando questionados sobre o setor em que trabalham, 32,6\% dos profissionais afirmaram atuar no setor de Raios X, seguido da Tomografia Computadorizada (25,5\%); setores de Densitometria Óssea, Mamografia ou Radiologia Odontológica com $21,4 \%$ e Radioterapia, com apenas $2,1 \%$.

A preponderância do sexo masculino nessa pesquisa está ancorada no perfil da própria classe profissional. Discute-se que a predominância do sexo masculino na área da radiologia está embasada nos padrões do sexismo, que consiste em qualquer atitude discriminativa fundamentada no sexo. Atitudes sexistas estão presentes na vida profissional de mulheres técnicas e tecnólogas em Radiologia. A falta de força física e a possibilidade de gravidez são alguns dos relatos mais frequentes quanto a não contratação de mulheres no setor de diagnóstico por imagem. Existe a crença de que o sexo feminino não é capaz de atuar na área da Radiologia por alguns equipamentos e setores demandarem muita força física. Além disso, por estarem diretamente expostas a radiação ionizante, quando as profissionais mulheres engravidam, há a necessidade de rápido afastamento das mesmas, fator considerado um grande prejuízo para os empregadores (Lins, 2018).

No que diz respeito ao setor de atuação dos profissionais entrevistados, é possível afirmar que a distribuição de equipamentos de imagem está diretamente associada a atuação dos profissionais das técnicas radiológicas. Em uma pesquisa realizada na Bahia, por Santos et al. (2019), os autores observaram que há um déficit na distribuição de equipamentos de imagem que empreguem maior tecnologia, como: Tomografia Computadorizada, Ressonância Magnética e Mamografia, ou seja, quanto maior a tecnologia do aparelho, menor a quantidade de aparelhos por habitante. Já o equipamento de Raios X convencional apresentou um superávit, por ter menor tecnologia e por ser um método de imagem mais básico, primordial e indispensável em estabelecimentos de saúde.

\subsection{Necessidade de capacitação e conhecimento em Libras pelos profissionais das técnicas radiológicas}

Na Tabela 2 constam as informações a respeito da oferta de capacitação em Libras pelo local de trabalho, como também sobre a existência de profissionais das técnicas radiológicas que saibam se comunicar em Libras no seu setor de atuação. 
Tabela 2 - Necessidade de capacitação e conhecimento em Libras pelos profissionais das técnicas radiológicas, N=82, Teresina-PI, 2021.

\begin{tabular}{|c|c|c|c|c|c|}
\hline & \multirow{2}{*}{ Variáveis } & \multirow[b]{2}{*}{$\mathbf{N}$} & \multirow[b]{2}{*}{$\%$} & \multicolumn{2}{|c|}{ Total } \\
\hline & & & & $\mathbf{N}$ & $\%$ \\
\hline \multirow{3}{*}{$\begin{array}{l}\text { Quanto a necessidade de ter } \\
\text { capacitação em Libras no seu } \\
\text { local de trabalho }\end{array}$} & $\begin{array}{l}\text { Sim, auxiliaria no atendimento dessa } \\
\text { clientela }\end{array}$ & 64 & 78 & \multirow{3}{*}{82} & \multirow{3}{*}{100} \\
\hline & $\begin{array}{l}\text { Não, o público é reduzido e não compensa } \\
\text { o investimento/tempo }\end{array}$ & 9 & 11 & & \\
\hline & $\begin{array}{l}\text { Não, acho mais eficiente ter disponível na } \\
\text { instituição uma pessoa capacitada em } \\
\text { Libras }\end{array}$ & 9 & 11 & & \\
\hline \multirow{4}{*}{$\begin{array}{l}\text { Em relação a existência de } \\
\text { algum profissional das } \\
\text { técnicas radiológicas que } \\
\text { saiba se comunicar em Libras }\end{array}$} & $\begin{array}{l}\text { Sim, se capacitou por estímulo da } \\
\text { instituição de trabalho }\end{array}$ & 1 & 1,2 & \multirow{4}{*}{82} & \multirow{4}{*}{100} \\
\hline & $\begin{array}{l}\text { Sim, se capacitou por conta própria para } \\
\text { fins profissionais }\end{array}$ & 9 & 11 & & \\
\hline & $\begin{array}{l}\text { Sim, domina a linguagem por motivos } \\
\text { pessoais }\end{array}$ & 3 & 3,6 & & \\
\hline & Não & 69 & 84,2 & & \\
\hline
\end{tabular}

Fonte: Autores (2021).

Dos entrevistados, $78 \%$ acreditam na necessidade da oferta de capacitação em Libras pelo local de trabalho. Entretanto, mesmo compreendendo a importância da capacitação em Língua Brasileira de Sinais, a maioria dos entrevistados $(84,2 \%)$ afirmam não existir profissional das técnicas radiológicas que saiba se comunicar em língua de sinais no seu setor/local de trabalho.

A necessidade da oferta de capacitação em Libras pelos locais de trabalho está fundamentada na expectativa, dos profissionais entrevistados, de oferecer um atendimento mais personalizado e adequado aos pacientes surdos. A capacitação obrigatória em Libras dentro do local de trabalho, e principalmente nos cursos da área da saúde, sem dúvidas proveria uma assistência mais humanizada, assertiva e digna ao paciente surdo, em função da melhoria da comunicação e troca de informações entre paciente e profissional da saúde, já que forneceria ao último o mínimo de entendimento necessário da Língua Brasileira de Sinais, necessária para o melhor atendimento da comunidade surda (Souza \& Porrozzi, 2009. Chaveiro et al., 2010).

Mesmo com a Lei Federal $n^{\circ} 10.436$ em vigor, que determina que as instituições públicas e empresas concessionárias de serviços públicos de assistência à saúde devam prestar assistência e atendimento adequado aos pacientes surdos, isso não ocorre, pois os profissionais da saúde não conhecem a língua de sinais, não há a oferta de cursos de capacitação, e também não há intérpretes de Libras no local hospitalar, dificultando ainda mais esse processo tão importante, e fazendo com que o profissional busque outros métodos para estabelecer a comunicação, mesmo que não sejam tão eficazes (Brasil, 2002. Cardoso, Rodrigues \& Bachion, 2006).

O fato de não haver profissionais capacitados na Língua de Sinais Brasileira em seus setores e/ou locais de trabalho pode acarretar em grandes prejuízos na saúde do paciente com surdez, pois a falta de treinamento e capacitação em Libras pode levar a diagnósticos errados e consequentemente tratamentos também. Ademais, a ineficiência da comunicação estabelecida pode fazer com que o paciente não siga a orientação dada pelo profissional, já que não há o entendimento do que foi dito. Mais 
uma vez reforça-se a importância de o profissional das técnicas radiológicas conhecer o mundo, cultura e língua da pessoa surda, para que eles possam vir a atender as necessidades comunicativas da comunidade surda, a partir do entendimento de ambos do que está sendo comunicado, gerando um sentimento de maior confiança e segurança tanto para o profissional quanto para o paciente (Magrini \& Santos, 2014. Pagliuca, Fiúza \& Rebouças, 2007).

\subsection{Processo comunicativo entre profissional das técnicas radiológicas e paciente surdo}

Os resultados acerca de como ocorre o processo de comunicação com pessoas surdas, destacando-se os desafios, estratégias, necessidade ou não de um intérprete, eficiência da comunicação e compreensão por parte do paciente, estão descritas no Gráfico 1 e Tabela 3.

Gráfico 1 - Identificação do intérprete que proporcionou auxílio aos profissionais das técnicas radiológicas durante realização de exames de imagem/terapias radiológicas, N=82, Teresina-PI, 2021.

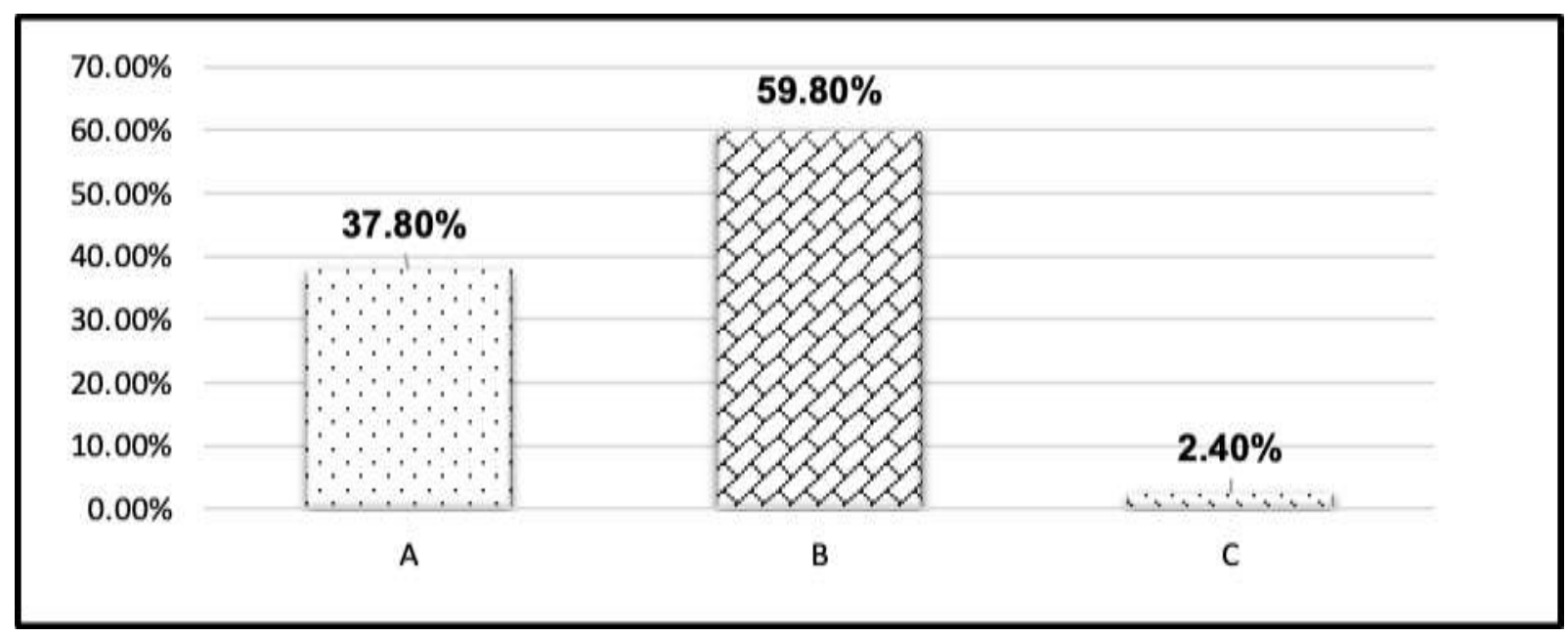

Legenda: A- Não foi necessário o auxílio de um intérprete para a realização do exame/terapia. B- Familiar que acompanhava o cliente surdo. C- Intérprete não familiar convidado ou contratado pelo cliente para acompanha-lo no exame/terapia. Fonte: Autores (2021).

Conforme o Gráfico 1, 59,8\% dos participantes apontaram que o intérprete foi um familiar do paciente que o acompanhava. Na tabela 3 há a caracterização do processo comunicativo entre profissional e paciente. O uso de gestos (43,1\%) foi o predominante quanto as estratégias de comunicação utilizadas. Além disso, 73,2\% dos profissionais afirmam que a comunicação foi eficiente e 80,5\% acreditam que a pessoa surda compreendeu a realização do exame de imagem a partir do método de comunicação utilizado. Por fim, 41,2\% dizem que esses exames e/ou terapias radiológicas com pessoas surdas costumam demandar mais tempo de execução. 
Tabela 3 - Processo comunicativo entre profissional das técnicas radiológicas e paciente surdo, N=82, Teresina-PI, 2021.

\begin{tabular}{|c|c|c|c|c|c|}
\hline \multirow{2}{*}{\multicolumn{2}{|c|}{ Variáveis }} & \multirow[b]{2}{*}{$\mathbf{N}$} & \multirow[b]{2}{*}{$\%$} & \multicolumn{2}{|c|}{ Total } \\
\hline & & & & $\mathbf{N}$ & $\%$ \\
\hline \multirow{6}{*}{$\begin{array}{l}\text { Quanto ao método de comunicação } \\
\text { utilizado para se comunicar com o } \\
\text { paciente surdo }\end{array}$} & Libras & 10 & 6,6 & \multirow{6}{*}{$151^{*}$} & \multirow{6}{*}{100} \\
\hline & Leitura Labial & 17 & 11,2 & & \\
\hline & Mímica & 34 & 22,5 & & \\
\hline & Gestos & 65 & 43,1 & & \\
\hline & Escrita & 13 & 8,6 & & \\
\hline & Intérprete & 12 & 8,0 & & \\
\hline \multirow{2}{*}{$\begin{array}{l}\text { Em relação a eficiência da } \\
\text { comunicação }\end{array}$} & Sim, foi eficiente & 60 & 73,2 & \multirow{2}{*}{82} & \multirow{2}{*}{100} \\
\hline & Não, não foi eficiente & 22 & 26,8 & & \\
\hline \multirow{2}{*}{$\begin{array}{l}\text { Quanto a compreensão do exame } \\
\text { de imagem por parte da pessoa } \\
\text { surda }\end{array}$} & Sim, acho que ela compreendeu & 66 & 80,5 & \multirow{2}{*}{82} & \multirow{2}{*}{100} \\
\hline & Não, acho que ela não compreendeu & 16 & 19,5 & & \\
\hline \multirow{5}{*}{$\begin{array}{l}\text { Em relação aos exames de } \\
\text { imagem/terapias radiológicas com } \\
\text { pacientes surdos, em geral eles se } \\
\text { caracterizam por }\end{array}$} & Demandam mais tempo de execução & 56 & 41,2 & \multirow{5}{*}{$136^{*}$} & \multirow{5}{*}{100} \\
\hline & $\begin{array}{l}\text { Necessidade de repetição do } \\
\text { exame/incidência }\end{array}$ & 24 & 17,6 & & \\
\hline & $\begin{array}{l}\text { Necessidade de acompanhante } \\
\text { durante o exame de imagem }\end{array}$ & 49 & 36,1 & & \\
\hline & $\begin{array}{l}\text { Necessidade de auxilio de mais } \\
\text { profissionais }\end{array}$ & 4 & 2,9 & & \\
\hline & $\begin{array}{l}\text { Necessidade de remarcar o exame de } \\
\text { imagem, para que o paciente traga um } \\
\text { acompanhante/interprete }\end{array}$ & 3 & 2,2 & & \\
\hline
\end{tabular}

$\mathrm{N}=151$ e N=136, pois havia a possibilidade de marcar mais de uma alternativa nas questões. Fonte: Autores (2021).

O auxílio do familiar, geralmente ouvinte, durante a realização de exames e terapias em geral tem como característica melhorar a relação entre paciente surdo e profissional da saúde. Já que esse acompanhante conhece a forma de se comunicar do surdo e todos os seus gestos e expressões, ele acaba por atuar como um intermediário e tradutor da fala do profissional para o paciente e vice-versa, melhorando a relação interpessoal dos mesmos (Cardoso, Rodrigues \& Bachion, 2006. Britto \& Samperiz, 2010).

Por assegurar a comunicação e o entendimento entre profissional e paciente surdo durante o procedimento radiológico, a presença dos acompanhantes/familiares acaba sendo uma prática comum durante o atendimento de saúde das pessoas surdas. Contudo, a estadia de uma terceira pessoa nesse atendimento, acaba por restringir a individualidade e autonomia do paciente surdo, já que quem explica e recebe as informações é o acompanhante, minimizando a oportunidade de a pessoa surda expor seus questionamentos (Oliveira, Celino \& Costa, 2015). 
Segundo Chaveiro, Barbosa \& Porto (2008, p. 501), "A barreira da comunicação que dificulta ou até impede a interação entre surdos e profissionais da saúde só pode ser superada quando se encontram formas de interação, única maneira de se garantir uma assistência de boa qualidade". A mímica, gestos e a leitura labial são métodos usados que atuam nesse sentido. Os movimentos, expressões faciais e gestos usados durante a mímica atuam de forma compensatória, já que são símbolos captados pela visão do surdo, similar ao uso de Libras.

Entretanto, como os símbolos usados na mímica não são universais, esse método de comunicação fica submetido a interpretação de quem o recebe. Já o uso de leitura labial também é possível nessa interação, mas pode ser facilmente inviabilizado por qualquer mudança na posição dos rostos do paciente surdo e profissional da saúde (Chaveiro, Barbosa \& Porto, 2008. Britto \& Samperiz, 2010).

A maioria dos profissionais das técnicas radiológicas entrevistados afirmam que a comunicação utilizada com o paciente surdo foi eficiente. Entretanto, mesmo utilizando de diversos métodos, como mímica, gestos e leitura labial, tais técnicas não garantem o tratamento correto, pois não são eficientes. A necessidade de comunicação das pessoas surdas excede a simples interpretação de gestos e mímicas realizadas pelos profissionais da saúde, já que existem outras diversas barreiras que tornam dificultoso a comunicação entre profissional-paciente. O pensamento, por esses profissionais, de que esses métodos são suficientes para atender bem o paciente surdo, apenas demonstra a falta de entendimento do amplo processo que são as necessidades comunicativas dessa parcela da população (Soares et al., 2018. Rezende, Guerra \& Carvalho, 2021).

Em uma pesquisa realizada por Souza et al. (2017), sobre os obstáculos enfrentados pela comunidade surda no atendimento de saúde, os resultados obtidos demonstram a falta de entendimento das pessoas surdas durante o atendimento de saúde. A pesquisa aponta que os pacientes surdos, na sua maioria, não entendem as informações e, devido a transmissão de informações ser unilateral (apenas o que o profissional da saúde quer expressar), não há comunicação eficiente, já que a compreensão pelo surdo não ocorre. Além disso, o estudo revela que não há, em serviços de saúde, pessoas capacitadas para se comunicar por Libras, sejam intérpretes disponíveis ou os próprios profissionais da saúde (Reis \& Santos, 2019).

A característica de maior demanda de tempo e execução dos exames de imagem foi bastante apontada pelos participantes. A mesma envolve os conceitos de produtividade e humanização, visto que devido a cobrança por realizar um determinado número desses exames por dia, os profissionais das técnicas radiológicas acabam esquecendo que antes de todo exame de imagem, existe um ser humano; e que o atendimento a eles deve ser individualizado e único, já que todos os pacientes são diferentes e possuem suas singularidades. Devido a isso, a humanização se faz importante, pois ela requer a prestação de um serviço de qualidade, baseado na escuta, vínculo e atenção ao paciente, fazendo com que o profissional enxergue além da patologia ou procedimento que deve ser realizado (Duarte \& Noro, 2013).

\section{Conclusão}

A partir desse estudo observou-se que a falta de capacitação advém da não oferta de cursos de Libras pelo local de trabalho. Além disso, os profissionais que buscaram capacitação, a minoria, o fez por conta própria e para fins profissionais. A não capacitação em língua de sinais pelos profissionais das técnicas radiológicas ocasiona um atendimento de saúde duvidoso e inferior ao que poderia ser ofertado caso esses profissionais tivessem conhecimento em Libras.

Ademais, verificou-se que os principais desafios impostos por esse relacionamento, são: falta de entendimento entre ambas as partes, falta de conhecimento em Libras, dificuldade em explicar etapas do procedimento e fazer com que o paciente obedeça aos comandos exigidos. Diante disso, métodos e estratégias são implementados e utilizados a fim de tentar estabelecer o mínimo de comunicação eficiente entre os pares, como: uso de gestos, mímica, leitura labial, escrita, Libras (pela minoria dos profissionais com entendimento básico) e auxílio de intérprete/acompanhante. O auxílio do acompanhante ocorreu na maioria dos atendimentos, já que geralmente é uma pessoa ouvinte e que também tem conhecimento na Língua de Sinais, 
fazendo a troca de informações entre profissional e paciente, permitindo que assim o exame de imagem e/ou terapia radiológica possa fluir melhor.

Já em relação ao nível de satisfação do próprio profissional com o seu atendimento, foi possível averiguar que, para a maioria, esse nível de satisfação está relacionado ao êxito na realização desses exames e terapias, ainda que ocorram desafios durante a explicação e execução dos mesmos. Entretanto, alguns profissionais demostraram insatisfação quanto ao seu atendimento, no que tange a falta de conhecimento em Libras e consequentemente, no impedimento de uma comunicação eficiente e adequada com o surdo.

Sendo assim, foi possível observar, identificar e entender quais desafios estão presentes nesse atendimento que carece de atenção e cuidados específicos, e também analisar a partir de qual momento e como ocorre a utilização de métodos e estratégias, para que possa ser oferecido o melhor atendimento possível, dentro das condições e limitações dos profissionais das técnicas radiológicas.

Diante dos resultados dessa pesquisa e visto que os trabalhos sobre esse tema são praticamente inexistentes, sugere-se para as pesquisas futuras: conhecer o ponto de vista da comunidade surda quanto ao atendimento de saúde realizado pelos profissionais das técnicas radiológicas, investigar a existência de profissionais intérpretes de Libras nos serviços de saúde e também a oferta da disciplina de Libras nos cursos de Técnico e Tecnólogo em Radiologia.

Por fim, espera-se que a oferta de capacitação obrigatória em Libras seja uma realidade nos locais de atendimento de saúde, e também que a disciplina de Língua Brasileira de Sinais seja ofertada de forma obrigatória em todos os cursos de saúde, para que assim, a inclusão social da comunidade surda seja plenamente alcançada nos serviços de saúde, oferecendo um serviço com clareza, confiança e respeito ao paciente, que independente das suas condições merece um atendimento da melhor qualidade possível.

\section{Referências}

Araujo, A.M. et al. (2019). A dificuldade no atendimento médico às pessoas surdas. Revista Interdisciplinar Ciências Médicas, 3(1), 3-9.

Brasil. (2002). Lei $n^{o}$ 10.436, de 24 de abril de 2002. Dispõe sobre a Língua Brasileira de Sinais - Libras e da outras providencias. Brasília. <http://www.planalto.gov.br/ccivil_03/leis/2002/110436.htm>

Britto, F. R. \& Samperiz, M. M. F. (2010). Dificuldades de comunicação e estratégias utilizadas pelos enfermeiros e sua equipe na assistência ao deficiente auditivo. Einstein, 8(1), 80-85.

Cardoso, A. H. A., Rodrigues, K.G. \& Bachion, M.M. (2006). Percepção da pessoa com surdez severa e/ou profunda acerca do processo de comunicação durante seu atendimento de saúde. Rev Latino-am Enfermagem, 14(4), 553-560.

Chaveiro, N., Barbosa, M. A. \& Porto, C. C. (2008). Revisão de literatura sobre o atendimento ao paciente surdo pelos profissionais da saúde. Revista da Escola de Enfermagem da USP, 42(3), 578-583.

Chaveiro, N. et al. (2010). Atendimento à pessoa surda que utiliza a língua de sinais, na perspectiva do profissional da saúde. Revista Cogitare Enfermagem, $15(4), 639-645$.

Duarte, M.L.C. \& Noro, A. (2013). Humanização do atendimento no setor de radiologia: dificuldades e sugestões dos profissionais de enfermagem. Revista Cogitare Enfermagem,18(3), 532-538.

Falqueto, J.M.Z, Farias, J.S. \& Hoffmann, V.E. (2018). Saturação teórica em pesquisas qualitativas: relato de uma experiência de aplicação em estudo na área de administração. Revista de Ciências da Administração, 20(52), 40-53.

Gatto, C.I. \& Tochetto, T.M. (2007). Deficiência auditiva infantil: implicações e soluções. Rev. CEFAC, 9(1), 110-115.

Lins, L. (2018). Os obstáculos que as mulheres enfrentam nas práticas radiológicas. <http://conter.gov.br/site/noticia/especial-4>

Magrini, A.M. \& Santos, T.M.M. (2014). Comunicação entre funcionários de uma unidade de saúde e pacientes surdos: um problema? Distúrb comum, 26(3), $550-558$.

Oliveira, Y.C.A., Celino, S.D.M. \& Costa, G.M.C. (2015). Comunicação como ferramenta essencial para assistência à saúde dos surdos. Revista Physis, 25(1), 307-320.

Pagliuca, L.M.F., Fiuza, N.L.G. \& Rebouças, C.B.A. (2007). Aspectos da comunicação da enfermeira com o deficiente auditivo. Rev. esc. enferm. USP, 41(3), 411-418. 
Research, Society and Development, v. 10, n. 17, e43101724222, 2021

(CC BY 4.0) | ISSN 2525-3409 | DOI: http://dx.doi.org/10.33448/rsd-v10i17.24222

Pereira, A. A. C. et al. (2020). "Meu Sonho É Ser Compreendido": Uma Análise da Interação Médico-Paciente Surdo durante Assistência à Saúde. Rev. bras. educ. med, 44(4), 1-9.

Prodanov, C.C. \& Freitas, E.C. (2013). Metodologia do Trabalho Científico: Métodos e Técnicas da Pesquisa e do Trabalho Acadêmico. Rio Grande do Sul: Feevale.

Reis, V. S. L. \& Santos, A. M. (2019). Knowledge and experience of Family Health Team professionals in providing healthcare for deaf people. Rev. CEFAC, 21(1), 1-8.

Rezende, R.F., Guerra, L.B. \& Carvalho, S.A.S. (2021). A perspectiva do paciente surdo acerca do atendimento à saúde. Revista CEFAC, 23(2), 1-10.

Santos et al. (2019). Distribuição de equipamentos de diagnóstico por imagem no âmbito do SUS: um panorama do estado da Bahia, Brasil. Rev. Bras. Pesq. Saúde, 21(4), 75-83.

Silva, M. L. et al. (2021). As dificuldades encontradas na assistência à saúde às pessoas com surdez. Research, Society and Development, 10(2), e38910212372.

Soares, I.P. et al. (2018). Como eu falo com você? A comunicação do enfermeiro com o usuário surdo. Revista Baiana de Enfermagem, 32 , e25978.

Souza, M. F. N. S. et al. (2017). Principais dificuldades e obstáculos enfrentados pela comunidade surda no acesso à saúde: uma revisão integrativa de literatura. Rev. CEFAC, 19(3), 395-405.

Souza, M.T. \& Porrozzi, R. (2009). Ensino de Libras para os profissionais da saúde: uma necessidade premente. Revista Práxis, 1(2), 43-46.

Tedesco, J. R. \& Junges, J. R. (2013). Desafios da prática do acolhimento de surdos na atenção primária. Cadernos de Saúde Pública, 29(8), 1685-1689. 